\title{
Altered Bone Remodeling in Psoriatic Arthritis
}

\author{
Kofi A. Mensah, M.S., Edward M. Schwarz, Ph.D., and Christopher T. Ritchlin, M.D. \\ The Center for Musculoskeletal Research, University of Rochester Medical Center, Rochester, New \\ York
}

\begin{abstract}
Bone is a highly dynamic organ that interacts with a wide array cells and tissues. Recent studies have unveiled unanticipated connections between the immune and skeletal systems and this relationship led to the development a new field, osteoimmunology. This field will enable investigators to translate basic science findings in bone biology to clinical applications for inflammatory joint diseases such as psoriatic arthritis (PsA). In this review, we will examine the disruption of bone homeostasis in PsA and discuss the pivotal role of osteoclasts and osteoblasts as well as signaling pathways in the altered remodeling observed in this inflammatory arthritis. We will also discuss the effects of TNF inhibition on both bone resorption and new bone formation in PsA.
\end{abstract}

\section{Keywords}

psoriasis; psoriatic arthritis; osteoclast; tumor necrosis factor; osteoblast; dickkopf-1

\section{Introduction}

At first glance, bone appears to be a static organ, simply there to provide structural support to the human form and to provide a niche for mesenchymal and hematopoietic progenitors. Bone, however, is a very dynamic organ as evidenced by the process of bone remodeling which relies on a delicate balance between bone formation and bone resorption, orchestrated by osteoblasts and osteoclasts respectively [1·]. The coordinated interplay of osteoblasts and osteoclasts continuously remodels bone through highly regulated molecular and cellular events such that the entire human skeleton is replaced over the course of each decade of life [2]. Disruption of the homeostatic balance of bone removal and replacement can manifest as pathologic bone loss observed in osteoporosis, periodontal disease, and some inflammatory arthritides or as inappropriate new bone formation found in spondyloarthritis $[1,3,4,5,6]$.

The dynamism of the skeleton is not limited to perpetual bone turnover but can also be observed in the interactions between bone and other organ systems. One particularly intriguing interaction which has gained much attention in recent years is the link between the skeletal and immunological systems. The recent understanding of an interplay between adaptive immune cells and cells involved in skeletal remodeling led to the development of a field known as osteoimmunology $[2,7,8]$. This rapidly expanding field has the potential to facilitate the translation of basic science knowledge in bone biology into an improved pathophysiological understanding of altered remodeling in inflammatory arthritis.

Corresponding Author: Edward M. Schwarz, Department of Orthopaedics, University of Rochester Medical Center, 601 Elmwood Avenue, Box 665, Rochester, NY 14642, Phone (585) 275-3063, Fax (585) 756-4721, E-mail: Edward_Schwarz@URMC.Rochester.Edu, Kofi_Mensah@urmc.rochester.edu, Christopher_Ritchlin@urmc.rochester.edu, (Honorarium to Kofi Mensah). 
In this review, we will discuss bone remodeling events as they relate to psoriatic arthritis (PsA), an inflammatory arthritis in which osteoimmune interactions can result not only in excess bone loss but also in pathologic new bone formation. Major factors involved in bone turnover, both established and under current investigation, such as tumor necrosis factor (TNF) and dickkopf-1 (DKK-1), will be discussed from the perspective of the altered bone remodeling observed in PsA. In particular, the effects that TNF exerts on the bone formation and function through its actions on osteoclasts and osteoblasts will be emphasized. Lastly, the impact of anti-TNF therapy on resorption of psoriatic bone coupled with the potential negative influence of these agents on the inhibition of pathological new bone formation characteristic of PsA will be examined.

\section{Osteoblasts and bone remodeling}

Osteoblasts are derived from pluripotent mesenchymal stem cells which can also give rise to chondrocytes, myoblasts, and adipocytes [7•.]. During the process of osteoblast differentiation, the pluripotent mesenchymal progenitors express higher quantities of phenotypic markers like alkaline phosphatase and osteocalcin. Mesenchymal progenitors also express receptors for bone morphogenetic proteins (BMP) and the Wnt receptors low-density lipoprotein receptor related proteins (LRP) 5 and 6, key receptors, which upon activation promote differentiation of these progenitors into bone-forming osteoblasts $[6,10]$.

Bone morphogenetic proteins, members of the TGF- $\beta$ superfamily, strongly regulate osteoblast differentiation [6]. BMPs bind two types of serine-threonine receptors which are both necessary for effective induction of a downstream signal cascade. Following binding of BMP to the BMP type I and BMP type II receptors, a protein family called Smads transduces and regulates the BMP signal cascade. Smad1 and Smad5 interact with the BMP receptor after BMP binds thereby leading to their activation. Smad4 then associates with and phosphorylates Smads1/5. Upon phosphorylation of Smad1/5, the whole complex is translocated to the nucleus where it regulates essential osteoblast differentiation via activation of transcription factors, like Cbfa1. Another molecule, Smad6, negatively regulates the signal cascade by competing with Smad1/5 for binding to BMP type I receptor. Smad6 also competes for binding of Smad4 to Smad1 $[6,9]$.

Another pathway that is a potent inducer of osteoblast differentiaton is signaling through Wnt [10]. The Wnt cascade is triggered when members of the Wnt class of proteins bind to a coreceptor complex which includes LRP 5 and 6 . These two receptors are indistinguishable in their ability to mediate Wnt signaling. Several downstream signaling proteins such as Disheveled are recruited by the intracellular domains LRP5/6 co-receptors. This protein is posttranslationally modified and then activates the canonical Wnt signaling cascade. Signaling through the Wnt cascade results in the stabilization of beta-catenin by preventing its degradation. When beta-catenin reaches high-enough levels in the cytoplasm, it translocates to the nucleus where it binds transcription factors to regulate expression of Wnt target genes $[10,11]$.

The important effects of the BMP-Smad and Wnt-LRP5/6 interactions on bone homeostasis stems from several in vivo and in vitro observations $[9,10]$. For example, transplantation of BMP into sites containing osteoprogenitors, like muscle or subcutaneous tissue, leads to ectopic bone formation, and LRP5 loss-of-function mutation leads to low bone mass while gain-offunction leads to the converse phenotype $[9,10]$. These two pathways have been shown to be centrally important in the generation of a mature osteoblast, which forms mineralized bone through the release of an osteoid matrix that hardens upon incorporation of calcium and phosphate. 


\section{Osteoclasts and bone remodeling}

Osteoclasts are multinucleated giant cells uniquely designed to resorb bone. Unlike their mesenchymal stem cell-derived osteoblast counterparts, osteoclasts are derived from hematopoietic cells in the monocyte-lineage. These hematopoietic-lineage cells also generate immune cells such as lymphocytes, phagocytes, and dendritic cells. Thus, osteoclasts derive from the same precursor as macrophages and myeloid dendritic cells [12]. The development of osteoclasts from their precursor cells has been studied by flow cytometric immunophenotyping of surface proteins. The multipotential myeloid progenitor cell population is defined as positive for the surface marker c-Kit. This population moderately expresses a pan-myeloid lineage marker CD11b, and is negative for c-Fms, which is the tyrosine kinase receptor for macrophage colony stimulating factor (M-CSF) — needed to prime cells for osteoclast differentiation. Upon interaction of these cells with stem cell factor (SCF), they become positive for the M-CSF receptor c-Fms [13]. C-Fms is a key determinant of development for cells in the monocyte-macrophage lineage [1.]. Thus, the multipotential progenitor cell is designated $\mathrm{c}-\mathrm{Kit}^{+} \mathrm{CD} 11 \mathrm{~b}^{\text {dull }} \mathrm{c}-\mathrm{Fms}^{-}$while the early-stage precursor is c$\mathrm{Kit}^{+} \mathrm{CD} 11 \mathrm{~b}^{\mathrm{dull}} \mathrm{c}-\mathrm{Fms}{ }^{+}$. The presence of M-CSF converts the early-stage precursor cells to latestage precursors by triggering increased $\mathrm{CD} 11 \mathrm{~b}$ expression and also by leading to upregulated surface expression of receptor-activator of NFKB (RANK) to which RANK ligand (RANKL) will bind in order to begin the cascade of signaling events which culminate in osteoclast formation [13].

RANKL is expressed by osteoblasts in the bone marrow stromal environment and this expression is induced in vivo by hormones like vitamin D3, parathyroid hormone, and estrogen [2,5]. In the absence of RANKL, the late-stage precursors will become macrophages. The osteoclasts, generated from late-stage precursors upon binding of RANKL, are mononuclear but a second event of major importance, multinucleation, takes place when mononuclear osteoclasts fuse with one another to form polykaryons $[5,13,14 \cdot]$. This process is analogous to the fusion events that take place between macrophages to form giant cells and requires the molecule dendritic cell-specific transmembrane protein (DC-STAMP). In support of the importance of this molecule in osteoclastogenesis are the findings that DC-STAMP-/- mice are osteopetrotic and they do not have multinucleated tartrate-resistant acid phosphatase (TRAP) osteoclasts $[15,16]$. Staining for TRAP is a histologic marker of osteoclasts and TRAP functions to decalcify bone when secreted through the osteoclast ruffled border at the resorption site. In addition to TRAP, osteoclasts acidify the local microenvironment on the bone surface by secreting $\mathrm{H}^{+}$ions, thereby mobilizing the mineral content of the bone. They then secrete cathepsin $\mathrm{K}$, which is involved in degradation of bone matrix exposed by the acid [1,18].

Osteoblasts are only one cell type capable of stimulating osteoclastogenesis via the osteoclastdifferentiating factor RANKL. Activated T-cells can also express membrane-bound and soluble RANKL and can thereby activate osteoclast development on RANK-expressing osteoclast precursors $[19,20]$. Mice deficient in RANKL are osteopetrotic because of the defect in osteoclastogenesis [21]. Transfer of T-cells that overexpress RANKL into RANKL-deficient mice can restore osteoclastogenesis [22]. Evidence for osteoimmunologic contributions to bone remodeling in inflammatory arthritis is shown by the findings that T-cell infiltrates coalesce in the synovial membranes of PsA patients, and that Th1 cytokines are present in high levels in PsA synovial fluid and tissue [23,24,25,26].

\section{Psoriatic arthritis: a disease of altered bone remodeling}

The major musculoskeletal signs of PsA can be defined as four entities: peripheral arthritis, axial disease, enthesitis, and dactylitis. These four musculoskeletal manifestations can present with varying degrees of altered bone remodeling in psoriatic bone and the combination of bone 
resorption and new bone formation is highly variable from patient to patient.. Not only is this variability noted between different patients but also within a patient in whom x-rays of a single digit can manifest both large eccentric erosions and digital ankylosis (Figure 1). The presence of joint erosion as part of the inflammatory process in the peripheral arthritis subset of PsA is found in 60-70\% of PsA patients [27]. In addition to joint erosion, a hallmark of spondyloarthropathies like PsA is the development of syndesmophytes and bony appositions in the digits that can lead to joint ankylosis [6,11]. Joint erosion and new bone formation (ankylosis, periostitis, syndesmophytes) can also be observed in patients with enthesitis, dactylitis and axial disease. Given the marked regional variation in altered bone remodeling, it is highly likely that the predominance of bone resorption or new bone formation at any given site of musculoskeletal pathology in PsA is governed by the local milieu where cytokines and growth factors exert effects on cells in the surrounding bony microenvironment.

\section{Altered bone resorption: TNF and osteoclasts}

The influence of the immune system on bone homeostasis is of central importance to understanding the pathologic alterations that underlie the bone resorption seen in PsA. In addition to M-CSF and RANKL, other cytokines upregulated during inflammation play a role in determining the fate of myeloid precursor cells capable of generating osteoclasts. Tumor necrosis factor (TNF) is one such cytokine, and in fact, RANKL is a member of the TNF family and has been called TNF-related activation-induced cytokine (TRANCE) [28]. TNF is an inflammatory cytokine produced by macrophages, neutrophils, keratinocytes, endothelial cells and fibroblasts with a myriad of effects from promoting cell-proliferation to facilitating apoptosis. Production of TNF is driven by NFKB response elements and its expression is largely regulated by post-transcriptional modifications of AU-rich elements (ARE) in the 3 ' region of the TNF gene. Resting cells have lower expression of TNF because the ARE mediate the degradation of TNF mRNA. After cellular activation, the TNF mRNA is stabilized and expression levels can increase 200-fold. Overexpression of TNF following cellular activation is prevented by the activity of tristetraprolin, which degrades the activation-induced TNF mRNA. Deregulation of the regulation of TNF expression following cellular activation can lead to chronically elevated TNF levels [29].

The link between deregulated TNF and inflammatory arthritis came out of observations that this cytokine is elevated in the synovial fluid and synovial membrane of rheumatoid arthritis and PsA patients [24]. In this context, TNF can cause joint inflammation and trigger cartilage destruction. Important to its role in altering bone remodeling is the pro-osteoclastogenic effect of TNF [30]. TNF can stimulate osteoclastogenesis via its interaction with the p55 subunit of the TNF receptor (TNFp55r) [30]. Upon binding to this receptor, TNF exerts several effects that foster increased osteoclast formation. TNF stimulates RANKL expression in bone marrow stromal cells and also activates the p38 MAPK cell-signaling pathway which leads to increased c-Fms expression. Binding of M-CSF to c-Fms stimulates RANK expression in osteoclast precursors. The RANKL upregulated by TNF in the bone marrow stromal cells binds to RANK on the osteoclast precursors and drives increased cell signaling downstream of RANK. A pivotal event in this signaling cascade is the activation of TRAF6, which is essential to osteoclastogenesis as TRAF6 knockout mice are osteopetrotic, and interferon-gamma has been demonstrated to halt osteoclast formation by targeting TRAF6 for degradation [4]. TRAF6

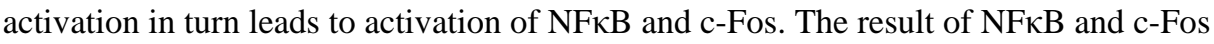
activation is the induction of NFATc1, a transcription factor, which leads ultimately to the increased expression of the genes for TRAP, cathepsin K, DC-STAMP and other genes essential for osteoclast formation and function.

In-vivo animal studies have also captured the importance of TNF in the development of autoimmune inflammatory erosive arthritis. The TNF-transgenic mouse, for example, closely 
mimics human disease and represents the first predictive animal model of arthritis as these animals develop erosive arthritis with focal subchondral and joint margin bone erosions [31]. On a cellular level, an effect of TNF in these animals is a four to seven-fold increase in the frequency of CD11 bi cells in peripheral tissues like spleen and blood that can serve as osteoclast precursors. The increase in this cell population coincided with the time at which TNF levels increased in these transgenic animals. Furthermore, treatment of the TNF transgenic mice with anti-TNF agents restored the number of cells in this population to levels seen in their wild type littermates [32]. In addition to the TNF transgenic model, an animal model for psoriasis and PsA also exists [33]. In this model, inducible epidermal deletion in JunB and cJun leads to phenotypic, histologic and immunohistochemical signatures of psoriasis and PsA. The inflammatory and erosive arthritis observed in this model is dependent on signaling through the TNF receptor.

\section{Altered bone formation: BMP, DKK-1, and osteoblasts}

Just as RANK-RANKL interactions are pivotal in osteoclastogenesis, BMP-BMPR interactions are key to osteoblastogenesis. Recent work has shown that perturbing the homeostasis of BMP signaling may play a direct role in joint ankylosis. Immunohistochemical analysis detected BMP and phosphorylated Smad1/5 in tissue sections of ankylosing enthesitits in a murine model of human spondyloarthropathy. Overexpression of a non-specific endogenous antagonist of BMP known as noggin led to decreased pathological severity in mice that develop ankylosis-like disease [6].

Wnt-LRP5/6 interactions are also central to osteoblastogenesis. Thus, blockade of the canonical Wnt signaling cascade leads to decreased bone formation. A natural antagonist of the canonical Wnt pathway is the glycoprotein dickkopf-1 (DKK-1). DKK-1 +/- mice have high bone mass and increased expression in transgenic mice leads to osteopenia [10]. It was recently shown that DKK-1 expression in inflammatory arthritis has two major consequences [11.•]. Increased DKK-1 expression impairs bone-forming osteoblast development and function by binding to the C-terminal domains of LRP5/6 receptors with high affinity thereby interfering with the Wnt-LRP5/6 stimulation of mesenchymal osteoblast precursors [10]. DKK-1 expression also suppresses the production of osteoprotegerin, a soluble receptor for RANKL that competes with RANK and inhibits activation of osteoclast precursors [34]. Taken together, DKK-1 favors osteoclastic bone resorption both by suppression of OPG and by inhibition of the bone reparative response.

\section{TNF and its effects (established and potential) in PsA}

The observation that TNF promotes bone resorption and inhibits new bone formation, coupled with its known effects on the frequency of osteoclast precursors, indicate that TNF is a pivotal cytokine in the pathophysiology of PsA. In support of this concept is the observation of elevated levels of TNF and soluble TNFp55r found in the sera, synovial fluid and synovial membranes of PsA patients [35]. Perhaps the most convincing evidence for the dominance of TNF in psoriatic joint inflammation and bone resorption arose from phase- 3 clinical trials which demonstrated a marked reduction in inflammation and progressive joint damage in subjects treated with anti-TNF agents compared to placebo discussed in detail below.

To elucidate the potential genetic basis for elevated TNF in PsA patients, the relationship between TNF promoter polymorphisms and PsA was evaluated in a study of 440 PsA patients and 204 controls. Of five polymorphisms analyzed, this study found a significant association between PsA and the -238 (A) polymorphism in the 5' flanking region of the TNF gene. A meta-analysis of data from six additional PsA cohorts strengthened the association between the -238 (A) TNF gene polymorphism and PsA with an overall odds ratio of 2.29 [36]. 
The relationship between elevated TNF and bone-resorbing osteoclasts in PsA is highlighted by a study of 24 PsA patients and 12 controls which showed significantly increased numbers of circulating, unstimulated osteoclast precursors derived from unstimulated cultured monocytes (i.e. no RANKL or M-CSF added to the cultures) in the PsA subjects relative to controls [37]. This study also found that higher numbers of osteoclast precursors were present in PsA patients with erosive disease evident on plain radiographs. The osteoclast precursor cells were determined to arise from the CD11 $b^{\text {hi }}$ peripheral blood mononuclear cell (PBMC) population; a finding similar to that observed in a study of a TNF transgenic arthritis mouse model by Li et al [32]. Blockade of TNF in the PsA patients brought the frequency of the $\mathrm{CD} 11 \mathrm{~b} \mathrm{bi}^{\mathrm{hi}}$ osteoclast precursor-containing population down to the levels of the healthy controls. Furthermore, the elevated TNF produced by PBMC isolated from PsA patients was able to induce healthy patient PBMC development into osteoclasts. This osteoclastogenic effect of the PsA PBMC-derived TNF was blocked by addition of anti-TNF agents [37]. A recent study also demonstrated that OCP frequency dropped dramatically following therapy with etanercept in patients with erosive PsA [38].

It was recently shown that TNF increases DKK-1 expression in inflammatory arthritis in the TNF transgenic mouse model. Data from this work showed a synergistic effect of TNF on the actions of DKK-1 is highlighted by the fact that blockade of both these molecules leads to a greater reduction in joint inflammation, bone erosion and osteoclast numbers per joint than blockade of DKK-1 alone. In terms of osteophyte formation, TNF blockade in the face of increasing concentrations of DKK-1 blockade did not show any difference relative to DKK-1 blockade alone in these animals. In both situations, increasing DKK-1 blockade resulted in increased osteophyte development [11..]. Another important mechanism by which TNF inhibits osteoblastic bone formation is by the induction of the E3-ubiquitin ligase Smurf1, which targets the critical osteoblast transcription factor Runx 2 for proteolysis [39]. Thus, TNF is a potent inhibitor of bone formation. These findings have implications for the efficacy of anti-TNF therapy in treating the dual alteration in bone remodeling of PsA.

\section{TNF blockade and altered bone remodeling in PsA}

TNF inhibitors have been used with unparalleled success in the treatment of PsA. Subjects on anti-TNF agents (etanercept, infliximab, adalimumab) demonstrated impressive clinical responses as measured by the ACR composite measure, HAQ and the PASI score. Given its role in stimulating osteoclast development, blockade of TNF should ameliorate the altered bone remodeling seen in PsA in addition to reducing inflammation. In a placebo-controlled phase-3 study using $25 \mathrm{mg}$ etanercept administered subcutaneously twice weekly, joint space narrowing and erosions were halted in the treatment group compared to the control group [40*-]. A phase-3 study of infliximab showed inhibition of radiographic disease progression at 6 months of treatment ref. A third anti-TNF agent approved for treatment of PsA is adalimumab, the fully human anti-TNF monoclonal antibody given subcutaneously at $40 \mathrm{mg}$ every other week or weekly. In a phase- 3 study of this agent, radiographic progression of disease as identified by hand and foot $\mathrm{X}$-rays was significantly inhibited [40*·].

Though bone resorption was halted by all three types of TNF inhibitors, phase- 3 clinical trial data for infliximab, etanercept and infliximab failed to demonstrate a decline in gross osteolysis, pencil-in-cup deformities or periostitis after six months of treatment which contrasts starkly with the ability of these agents to inhibit structural damage at the same timepoint. It has been suggested that continued suppression of inflammation via anti-TNF agents may accelerate new bone formation and ankylosis [6]. The role of TNF in upregulating DKK-1 expression may help explain this observation. Serum DKK-1 levels in patients with RA decreased over the course of six weeks of anti-TNF therapy. The serum DKK-1 levels in untreated patients with ankylosing spondylitis, were significantly lower than that of untreated 
healthy controls $(\sim 5 \mathrm{pg} / \mathrm{mL}$ versus $\sim 15 \mathrm{pg} / \mathrm{mL})[11 \cdot \cdot$. Thus, TNF blockade decreases the inhibitory potential of DKK-1 on the pro-osteoblastogenic Wnt signaling pathway. Thus patients with ankylosing spondylitis and possibly a subset of PsA patients may have accelerated pathologic new bone formation when treated with anti-TNF agents due lower DKK-1 levels and subsequent disinhibition of Wnt signaling. Indeed, the inability of TNF inhibition to halt bony progression was recently demonstrated in phase three trials of ankylosing spondylitis [41]. Anti-TNF agents may also not be effective in the amelioration of new bone formation pathology in PsA because they may not target the inappropriately activated BMP pathway thought to play a role in the development of ankylosis and enthesitis as studies with an ankylosing spondylitis mouse model demonstrated that joint inflammation was not coupled to pathologic bone formation [6,42]. Perhaps, therapies aimed at the Wnt and BMP signaling cascades would be helpful adjuncts to anti-TNF therapy in the treatment PsA patients with a phenotype characterized by widespread new bone formation.

\section{Conclusions}

Musculoskeletal inflammation is a common feature of psoriasis and can manifest radiographically as bone loss or new bone formation. Indeed the recently published CASPAR study included radiographically identifiable joint damage as part of the diagnostic criteria [43]. The alterations in bone remodeling seen in PsA are the result of disruption in the careful regulation of bone homeostasis. Central to deregulated bone turnover are the functions of boneeroding osteoclasts and bone-forming osteoblasts. The osteoimmune interface in PsA also involves the potentiation of RANK-RANKL signaling by TNF, a potent pro-inflammatory cytokine elevated in PsA where a significant correlation between disease activity and mutations in the TNF gene was observed. Elevated TNF not only potentiates signaling in osteoclast precursors, but it also increases the number of cells capable of becoming such precursors. Furthermore, TNF can affect the other half of the normally balanced bone remodeling process by inducing DKK-1 to inhibit bone-forming osteoblast development via inhibition of Wnt signaling. The remarkable success of anti-TNF agents in the treatment of PsA is not only a result of their ability to reduce inflammation, but also because of their ability to prevent further deterioration of bone by mitigating osteoclast-mediated erosion of the joints. Despite this, the effect of DKK-1 and the inappropriate activation of the BMP signaling pathway on osteoblastogenesis are areas where anti-TNF agents may not provide as much benefit in PsA and may actually worsen new bone formation. Future studies on altered bone remodeling in this disease may further elucidate the mechanisms of new bone formation, particularly the levels of activation for BMP and DKK-1. Future studies may also be aimed at uncovering new therapeutic targets, perhaps the Smads or Wnt signaling, that may act together with the anti$\mathrm{TNF}$ agents to restore the dynamic balance between erosion and formation in psoriatic bone.

\section{Acknowledgments}

The authors are supported by research grants for the US Dept. of Defense (ERMS No.06136016 and UID No. 99853) and the National Institutes of Health PHS awards AR48681, DE17096, AR46545, AR54041 and UL1 RR24160 and the National Psoriasis Foundation.

\section{References}

1. Teitelbaum SL. Osteoclasts: what do they do and how do they do it? Am J Pathol 2007;170:427-435. [PubMed: 17255310]A good review of recent advances in osteoclast biology summarizing their formation and function as well as key regulators in these processes.

2. Rho J, Takami M, Choi Y. Osteoimmunology: interactions of the immune and skeletal systems. Mol Cells 2004;17:1-9. [PubMed: 15055519]

3. Rodan GA, Martin TJ. Therapeutic approaches to bone disease. Science 2000;289:1508-1514. [PubMed: 10968781] 
4. Takayanagi H, Ogasawara K, Hida S, et al. T-cell-mediated regulation of osteoclastogenesis by signaling crosstalk between RANKL and IFN-gamma. Nature 2000;408:600-605. [PubMed: 11117749]

5. Boyle WJ, Simonet WS, Lacey DL. Osteoclast differentiation and activation. Nature 2003;423:337342. [PubMed: 12748652]

6. Lories RJU, Derese I, Luyten FP. Modulation of bone morphogenetic protein signaling inhibits the onset and progression of ankylosing enthesitis. J Clin Invest 2005;115:1571-1579. [PubMed: 15902307]

7. Walsh MC, Kim N, Kadono Y, et al. Osteoimmunology: interplay between the immune system and bone metabolism. Annu Rev Immunol 2006;24:33-63. [PubMed: 16551243]An excellent review of this new and upcoming field. This article covers aspects of bone biology as well as the recent advancements in understanding the links between adaptive immune cells, particularly T-cells, and bone homeostasis.

8. Takayanagi H. Osteoimmunology: shared mechanisms and crosstalk between the immune and bone systems. Nat Rev Immunol 2007;7:292-304. [PubMed: 17380158]

9. Yamaguchi A, Komori T, Suda T. Reulation of osteoblast differentiation mediated by bone morphogenetic proteins, hedgehogs and Cbfa1. Endocrine Reviews 2000;21:393-411. [PubMed: 10950158]

10. Baron R, Rawadi G. Minireview: Targeting the Wnt/B-Catenin pathway to regulate bone formation in the adult skeleton. Endocrinology 2007;148:2635-2643. [PubMed: 17395698]

11. Diarra D, Stolina M, Polzer K, et al. Dickkopf-1 is a master regulator of joint remodeling. Nat Medicine 2007;13:156-163.This article describes DKK-1 as a potential marker of joint remodeling and its position in the bone turnover axis. The article includes data from animal models and patients with inflammatory arthritis and provides insight as to another mechanism of TNF's influence on bone remodeling.

12. Miyamoto T, Ohneda O, Arai F, et al. Bifurcation of osteoclasts and dendritic cells from common progenitors. Blood 2001;98:2544-2554. [PubMed: 11588053]

13. Arai F, Miyamoto T, Ohneda O, et al. Commitment and Differentiation of Osteoclast Precursor Cells by the Sequential Expression of c-Fms and Receptor Activator of Nuclear Factor \{kappa\}B (RANK) Receptors. J Exp Med 1999;190:1741-1754. [PubMed: 10601350]

14. Vignery A. Macrophage fusion: the making of osteoclasts and giant cells. J Exp Med 2005;202:337340. [PubMed: 16061722]A good summary of the current models for the role of a relatively new protein, DC-STAMP, in the osteoclast fusion process.

15. Kukita T, Wada N, Kukita A, et al. RANKL-induced DC-STAMP is essential for osteoclastogenesis. J Exp Med 2004;200:941-946. [PubMed: 15452179]

16. Yagi M, Miyamoto T, Sawatani Y, et al. DC-STAMP is essential for cell-cell fusion in osteoclasts and foreign body giant cells. J Exp Med 2005;202:345-351. [PubMed: 16061724]

17. Roodman GD. Advances in bone biology, the osteoclast. Endocr Rev 1996;17:308-332. [PubMed: 8854048]

18. Teitelbaum SL. Bone resorption by osteoclasts. Science 2000;289:1504-1508. [PubMed: 10968780]

19. Wong BR, Rho J, Arron J, et al. TRANCE is a novel ligand of the tumor necrosis factor receptor family that activates c-Jun N-terminal kinase in T-cells. J Biol Chem 1997;272:25190-25194. [PubMed: 9312132]

20. Anderson DM, Maraskovsky E, Billingsley WL, et al. A homologue of the TNF receptor and its ligand enhance T-cell growth and dendritic-cell function. Nature 1997;390:175-179. [PubMed: 9367155]

21. Kong YY, Feige U, Sarosi I, et al. Activated T-cells regulate bone loss and joint destruction in adjuvant arthirits through osteoprotegerin ligand. Nature 1999;402:304-309. [PubMed: 10580503]

22. Kim N, Odgren PR, Kim DK, et al. Diverse roles of the tumor necrosis factor family member TRANCE in skeletal physiology revealed by TRANCE deficiency and partial rescue by a lymphocyte-expressed TRANCE transgene. Proc Natl Acad Sci USA 2000;97:10905-10910. [PubMed: 10984520]

23. Fitzgerald $\mathrm{O}$. Advances in understanding and novel therapeutic targets in inflammatory arthritis. Ir J Med Sci 1995;164:4-11. [PubMed: 7890533] 
24. Ritchlin C, Haas-Smith SA, Hicks D, et al. Patterns of cytokine production in psoriatic synovium. J Rheumatol 1998;25:1544-1552. [PubMed: 9712099]

25. Partsch G, Steiner G, Leeb BF, et al. Highly increased levels of tumor necrosis factor-a and other proinflammatory cytokines in psoriatic arthritis synovial fluid. J Rheumatol 1997;24:518-523. [PubMed: 9058659]

26. McInnes IB, Illei GG, Danning CL. IL-10 Improves Skin Disease and Modulates Endothelial Activation and Leukocyte Effector Function in Patients with Psoriatic Arthritis. J. Immunol 2001;167:4075-4082. [PubMed: 11564829]

27. Gladman DD, Farewell VT, Nadeau C, et al. Clinical indicators of progression in psoriatic arthritis: multivariate relative risk model. J Rheumatol 1995;22:675-679. [PubMed: 7791162]

28. Suda T, Takahashi N, Udagawa N, et al. Modulation of Osteoclast Differentiation and Function by the New Members of the Tumor Necrosis Factor Receptor and Ligand Families. Endocr Rev 1999;20:345-357. [PubMed: 10368775]

29. Li P, Schwarz EM. The TNF-alpha transgenic mouse model of inflammatory arthritis. Springer Semin Immunopathol 2003;25:19-33. [PubMed: 12904889]

30. Abu-Amer Y, Erdmann J, Kollias G, et al. Tumor necrosis factor receptors types 1 and 2 differentially regulate osteoclastogenesis. J Biol Chem 2000;275:27307-27310. [PubMed: 10874036]

31. Keffer J, Probert L, Cazlaris H, et al. Transgenic mice expressing human tumour necrosis factor: a predictive genetic model of arthritis. EMBO J 1991;10:4025-4031. [PubMed: 1721867]

32. Li P, Schwarz EM, O'Keefe RJ, et al. Systemic tumor necrosis factor alpha mediates an increase in peripheral CD11bhigh osteoclast precursors in tumor necrosis factor alpha-transgenic mice. Arthritis Rheum 2004;50:265-276. [PubMed: 14730625]

33. Zenz R, Eferl R, Kenner L, et al. Psoriasis-like skin disease and arthritis caused by inducible epidermal deletion of Jun proteins 2005;437:369-375.

34. Simonet WS, Lacey DL, Dunstan CR, et al. Ostoeprotegerin: a novel secreted protein involved in the regulation of bone density. Cell 1997;89:309-319. [PubMed: 9108485]

35. Mease PJ. Tumor necrosis factor (TNF) in psoriatic arthritis: pathophysiology and treatment with TNF inhibitors. Ann Rheum Dis 2002;61:298-304. [PubMed: 11874829]

36. Rahman P, Siannis F, Butt C, et al. TNFa polymorphisms and risk of psoriatic arthritis. Ann Rheum Dis 2006;65:919-923. [PubMed: 16284098]

37. Ritchlin CT, Haas-Smith S, Li P, et al. Mechanisms of TNF-a- and RANKL-mediated osteoclastogenesis and bone resorption in psoriatic arthritis. J Clin Invest 2003;111:821-831. [PubMed: 12639988]

38. Anandarajah AP, Schwarz EM, Totterman S, et al. The effect of etanercept on osteoclast precursor frequency and enhancing bone marrow oedema in patients with psoriatic arthritis. Ann Rheum Dis 2008;67:296-301. [PubMed: 17967829]

39. Kaneki H, Guo R, Chen D, et al. Tumor necrosis factor promotes runx 2 degradation through upregulation of smurf1 and smurf2 in osteoblasts. J Biol Chem 2006;281:4326-4333. [PubMed: 16373342]

40. Mease PJ. Psoriatic arthritis update. Bull NYU Hosp Jt Dis 2006;64:25-31. [PubMed: 17121486]A nice review of the TNF-inhibitor clinical trial data and their findings related to PsA. Provides insight as to the effectiveness of anti-TNF agents on limiting bone resorption as well as calling to mind concerns about the ability of these drugs to halt new bone formaiton.

41. Van der Heijde DM, Landewe RD, Ory P, et al. Etanercept does not inhibit radiographic progression in patients with ankylosing spondylitis. Ann Rheum Dis 2006;65(SII):81. [PubMed: 15919677] (abstract)

42. Lories RJ, Derese I, Bari C, et al. Evidence for uncoupling of inflammation and joint remodeling in a mouse model of spondylarthritis. Arthritis Rheum 2007;56:489-497. [PubMed: 17265484]

43. Taylor W, Gladman D, Helliwell P, et al. Classification criteria for psoriatic arthritis: development of new criteria from a large international study. Arthritis Rheum 2006;54:2665-2673. [PubMed: 16871531] 


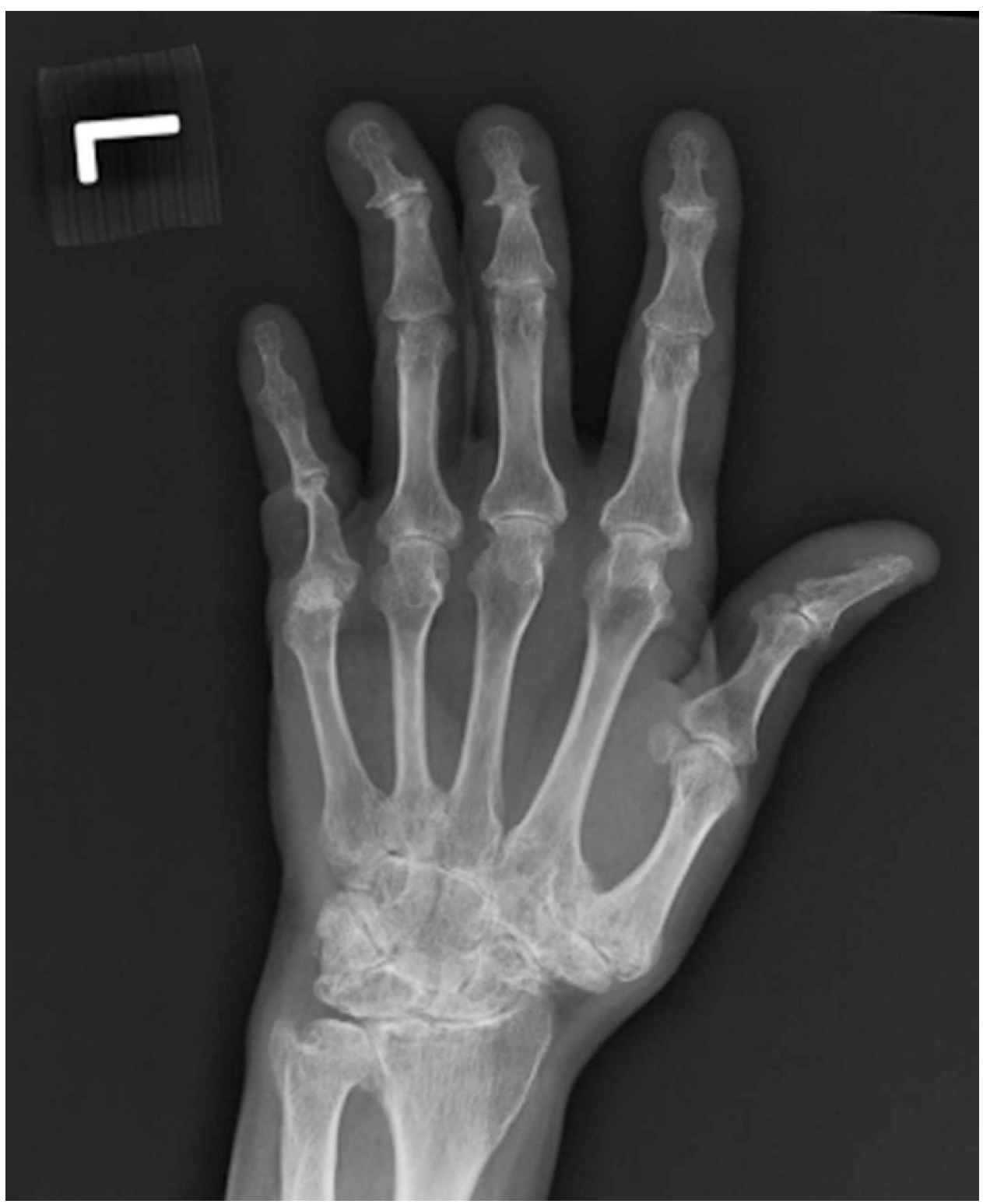

Figure 1. Bone formation and resorption in PsA hand

Note the ankylosis of the second and fifth distal interphalangeal joints and contrast with the marked erosive changes in the wrists and the second, third and fifth metacarpal phalangeal joints. 